\title{
Removing a barrier - the effect of plant-based cooking skills on dietary habits. A mixed-method study
}

\author{
Malou Reipurth, Charlotte G. Gregersen and Federico J. Armando Perez-Cueto \\ University of Copenhagen, Copenhagen, Denmark
}

\section{Abstract}

Previous reports have shown that people perceive lack of cooking skills as a barrier to adopting a plant-based diet (Reipurth et al., 2018; Klöckner, 2017; Haverstock and Forgays, 2012; Pohjolainen, Vinnari, and Jokinen, 2015; Mullee et al., 2017). Cooking skills have been defined as "a set of mechanical or physical skills used in meal preparation" by Ternier (2010), whereas some perceive them as more complex and abstract, involving ideas, knowledge, and planning skills (Short, 2003; Caraher, 1999). This mixed-method study was conducted with the aim of investigating the impact of cooking skills as a barrier, and which effect applied cooking skills have on consumers adopting and sustaining a plant-based diet. This was done by looking at people who had gained plant-based cooking skills by receiving a product that educated them in plant-based foods and delivered recipes and ingredients, with which the recipients cooked plant-based meals. The mixed-method study was comprised of a qualitative part (two focus group interviews with 10 participants) and a quantitative part (an online survey of 303 respondents).

The survey respondents were segmented based on their change in dietary habits after receiving the products, which was measured by their recalled change in intake of meat, fish, dairy, eggs, and plant-based foods. Four segments were identified; "Same All", "Lower Omni Higher Plant", "Lower Meat Higher Plant", and "Lower Meat". Clusters were profiled applying logistic regression models with cluster membership as the dependent variable, whereas the independent variables were the 12 attitudes towards consumption of plantbased diets, and the reasons for buying the product (also referred to as motivations), respectively.

The results from the qualitative study showed that the participants experienced minor changes while receiving the product, but lacked the menu planning skills, convenience, and habits necessary to maintain their plant-based diets after-wards. The results from the quantitative study suggested that changes towards plant-based consumption are affected by ethical factors, health, and sensory characteristics. A main finding of the quantitative survey was that it demonstrated how applying new cooking skills and experience with plant-based meals increase consumers' intake of plant-based food and decrease their intake of animal products. This effect was greatest while receiving the product, although still evident up to six months after. The findings from the study concur with and add more depth to the findings from preceding literature on the subject and can be used to inform and inspire research towards promoting plant-based diets.

\section{Conflict of Interest}

There is no conflict of interest 